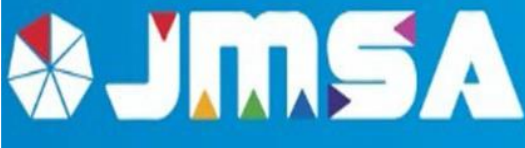

\section{The Dependence of the Perspective Illuminance Distribution on the Roadway Surface on the Luminaire Cross Factor (CF)}

\author{
F. M. El- Sharkawy", A. Al Kamel and M.M. El-Ganainy \\ National Instituted of Standard, Photometry, Giza, Egypt \\ Corresponding author e-mail: "fsharkawy_2000@yahoo.com.
}

Received on: 3 March 2021; Accepted on 26 April 2021.

\begin{abstract}
In this work a mathematical equation has been derived to express the relative illuminance at a point $\mathrm{P}$ on the road surface $\varepsilon(\mathrm{P})$ as a function of luminaire mounting height $(\mathrm{h})$, the indices of the luminaire lighting distribution $(\mathrm{mt})$ across, $(\mathrm{ml})$ along the road surface and the Cartesian coordinates of the point $(\mathrm{x}, \mathrm{y})$. For each value of the luminaire $\mathrm{CF}$, the equation represents the geometrical locus of the point $\mathrm{P}$ having a certain value of $\varepsilon(\mathrm{P})$ (isolux contours). To take advantage of such equations the calculation of $\varepsilon(\mathrm{P})$ aimed to realize the two following technical points. Firstly, i) Drawing the isolux diagram corresponding to the case at which $\mathrm{CF}=10$ and $\mathrm{H}=10 \mathrm{~m}$. ii) Calculating $\varepsilon(\mathrm{P})$ and the relative luminance $\mathrm{L}^{\prime}(\mathrm{P})$ at the point $\mathrm{P}$ corresponding to different values of CF for the standard road surface R2. Secondly, i) -Obtaining the isolux diagram features corresponding to different values of $\mathrm{CF}$. ii) The obtained values of the isolux diagram features allow us to readily comprehend the distribution of illuminance, and luminance over the road surfaces. According to the definition of both the absolute illuminance and luminance uniformities, the two quantities are equaled to the minimum values of $\varepsilon(\mathrm{P})$ and $\mathrm{L}^{\prime}(\mathrm{P})$ respectively. This enables determining the quality of the lighting systems.
\end{abstract}

Keywords: Illuminance; Luminance; Luminaire cross factor.

\section{Introduction}

\subsection{Illuminance and luminance approach in road lighting}

The roadway lighting committee of Illuminating Engineering Society's (IES) is considering the possible basis of specifying roadway lighting. It has been found that in order to produce ideal luminaire distributions to get perfect uniformity only on an illumination basis for vertical, horizontal or at any other measurement planes [1]. The C.I.E., recommendation for lighting the roads for motorized traffic specifies the luminance approach. A further edition of the IES lighting handbook [2] at which the luminance and illuminance are inter related. The approach of illuminance is important for the analysis of the geometrical relationship between the roadways, and the lighting installation procedure such as: 
1) Arrangements with variable mounting height, spacing overhang, and roadway width.

2) Uniformity of downward luminous flux;

3) Evaluation of luminous flux utilization factor and;

4) The cost associated with the installation operation and maintenance of upgrading lighting installation.

\subsection{The lighting distribution of the luminaire}

The lighting distribution (LD) of a luminaire is the basic information in any lighting calculation. Usually, the needed lighting calculations are that mainly used for drawing isolux diagrams, assessment of luminous flux, and output ratio. There is a convenient means of describing (LD) known as intensity distribution solid [3]. It is a portion of space bounded by a surface constituting a geometrical locus of the endpoint of the position vectors that represent the luminous intensity in different directions. Depending on the shape of the intensity distribution solid, one can break all sources down into two groups; symmetrical and non-symmetrical.

\subsection{Luminaire CF [4]}

Van Bommel derived the so-called luminaire $\mathrm{CF}$ with which it is possible to describe the suitability for use under the wet condition of any luminaire. He showed that CF could be defined as the intensity ratio $\boldsymbol{I}_{t} / \boldsymbol{I}_{l}$ where $\boldsymbol{I}_{t}$ is the luminous intensity of the luminaire in the direction $45^{\circ}\left(\tan ^{-1} 1\right)$ from the downward vertical in the plane at a right angle to the road axis. $\boldsymbol{I}_{l}$ : the luminous intensity of the luminaire in the direction $63^{\circ} .5\left(\tan ^{-1} 2\right)$ from the downward vertical in the plane parallel to the road axis. The result of calculations showed the increase in overall uniformity with an increase in $\mathbf{C F}$ for three types of the wet road surface.

\subsection{The absolute illuminance and luminance uniformities}

It is well known that the uniformity of both illuminance and luminance on the road surface is one of the quality parameters which determine the visual performance of the road lighting. There are different formulae for uniformity assessment. In this work, we are dealing with the absolute illuminance and luminance uniformities. The absolute illuminance uniformity is defined as the ratio of the minimum to the maximum illuminance on the road surface. The absolute luminance uniformity is defined as the ratio of the minimum to the maximum luminance on the road surface [4].

\section{Theoretical}

\subsection{The determination of the relative illuminance at any point on the road surface}

Considering the typical layout Figure (1). Taking the point $\mathrm{O}$ as the origin of the coordinates directly below the symmetrical luminaire of mounting height $\mathrm{h}, \mathrm{x}$ and $\mathrm{y}$ as the axis of coordinates, then (x-y) plane represents the road surface. 


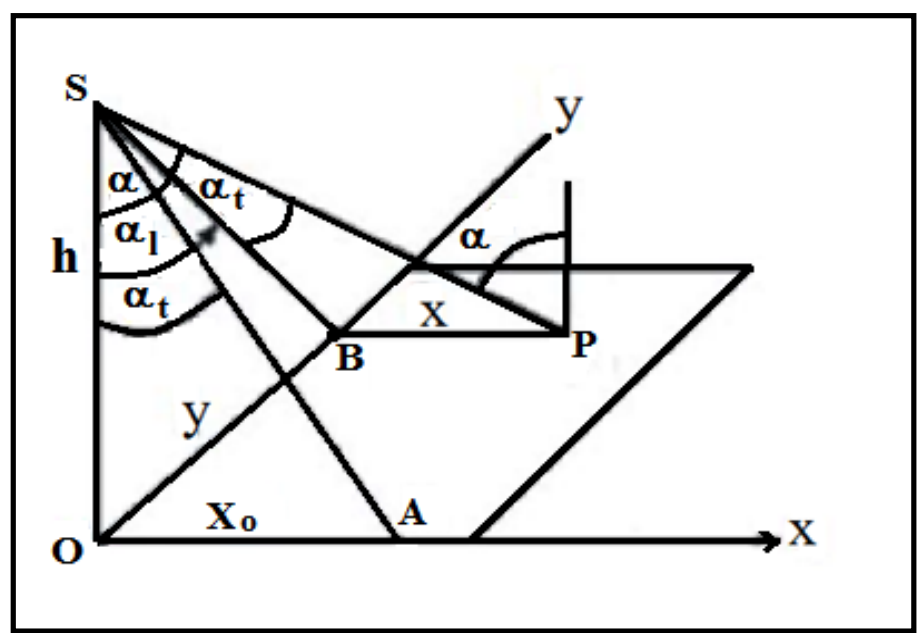

Fig. 1. The localization of the point $\mathrm{P}$ on the road surface.

It was found that the most suitable analytical expressions for luminous intensity $\left(I_{\alpha}\right)$, and the illuminance $\left(\boldsymbol{E}_{\boldsymbol{P}}\right)$ versus the angle between the axis of symmetry, and the direction of the beam are expressed by the two following formulae [5].

$$
\begin{aligned}
& \mathrm{I}_{\alpha}=\mathrm{I}_{\mathrm{o}}(\cos \alpha)^{\mathrm{m}} \\
& \mathrm{E}_{\mathrm{P}}=\mathrm{E}_{\mathrm{o}}(\cos \alpha)^{\mathrm{m}+3}
\end{aligned}
$$

and

Where $I_{o}$ is the value of the intensity in the direction of the axis of symmetry, $\mathbf{E}_{\mathbf{o}}=\frac{\mathbf{I}_{\mathbf{o}}}{\mathbf{h}^{\mathbf{2}}}$ and $\mathbf{m}$ is the index of the luminaire lighting distribution. Hence, the relative illuminance

$$
\varepsilon(P)=\frac{E_{P}}{E_{0}}=(\cos \alpha)^{m+3}
$$

Assuming the three points $\boldsymbol{A}, \boldsymbol{B}$, and $\boldsymbol{P}$, in Figure (1) representing the coordinate (x, $0),(0, y)$ and $(\mathrm{x}, \mathrm{y})$ respectively.

Let the relative illuminance at the three points be denoted by $\varepsilon(\mathbf{A}), \boldsymbol{\varepsilon}(\mathbf{B})$, and $\boldsymbol{\varepsilon}(\mathbf{P})$. Considering the fact of symmetry, the dependence of $\boldsymbol{\varepsilon ( P )}$ on $\boldsymbol{\varepsilon}(\mathbf{A})$ and $\boldsymbol{\varepsilon}(\mathbf{B})$ is given by the formula [5].

$$
\varepsilon(\mathrm{P})=\varepsilon(\mathrm{A}) \cdot \varepsilon(\mathrm{B})
$$

Where:

$$
\varepsilon(A)=\left(\cos \alpha_{t}\right)^{m_{t}+3}, \varepsilon(B)=\left(\cos \alpha_{l}\right)^{m_{l}+3}, \text { and } \varepsilon(P)=(\cos \alpha)^{m+3}
$$

Where $\boldsymbol{m}_{t}, \boldsymbol{m}_{l}$, and $\boldsymbol{m}$ are the indices of L.D in the direction of the beams incident at the three points respectively.

The angles are illustrated in Figure (1). Considering the fact of symmetry, the angle between the two beams incident at points $\boldsymbol{B}$ and $\boldsymbol{P}$ equals to $\boldsymbol{\alpha}_{\boldsymbol{t}}$. Then we get the following equation:

$$
\cos \alpha_{\mathrm{t}}=\frac{\mathrm{h}}{\sqrt{\mathrm{h}^{2}+\mathrm{x}_{0}^{2}}}=\frac{\sqrt{\mathrm{h}^{2}+\mathrm{y}^{2}}}{\sqrt{\mathrm{h}^{2}+\mathrm{x}^{2}+\mathrm{y}^{2}}}, \cos \alpha_{1}=\frac{\mathrm{h}}{\sqrt{\mathrm{h}^{2}+\mathrm{y}^{2}}}, \cos \alpha=\frac{\mathrm{h}}{\sqrt{\mathrm{h}^{2}+\mathrm{x}^{2}+\mathrm{y}^{2}}}
$$

By substituting for, $\cos \boldsymbol{\alpha}_{\mathbf{t}}$ and $\cos \boldsymbol{\alpha}_{\mathbf{l}}$ from equation (6) in equation (5), and for $\boldsymbol{\varepsilon}(\mathbf{A})$, $\boldsymbol{\varepsilon}(\mathbf{B})$ and $\boldsymbol{\varepsilon}(\mathbf{P})$ from Equation (5) in Equation (4) get:

$$
\varepsilon(\mathbf{P})=\left(\frac{\mathrm{h}}{\sqrt{\mathrm{h}^{2}+\mathrm{x}^{2}+\mathrm{y}^{2}}}\right)^{m+3}=\left(\frac{\sqrt{\mathrm{h}^{2}+\mathrm{y}^{2}}}{\sqrt{\mathrm{h}^{2}+\mathrm{x}^{2}+\mathrm{y}^{2}}}\right)^{m_{t}+3} \cdot\left(\frac{\mathrm{h}}{\sqrt{\mathrm{h}^{2}+\mathrm{y}^{2}}}\right)^{m_{l}+3}
$$


Equation (7) shows the dependence on the $\mathbf{x}, \mathbf{y}$ coordinates of the point $\boldsymbol{p}$ on the road surface, the luminaire mounting height $(\boldsymbol{h})$, and the indices $\boldsymbol{m}_{t}$ and $\boldsymbol{m}_{l}$ of the luminaire L. D.

Putting: $\quad \mathbf{F}=\frac{\sqrt{\mathbf{h}^{2}+\mathbf{y}^{2}}}{\sqrt{\mathbf{h}^{2}+\mathbf{x}^{2}+\mathbf{y}^{2}}}, \quad$ and $\quad \mathbf{G}=\frac{\mathbf{h}}{\sqrt{\mathbf{h}^{2}+\mathbf{y}^{2}}}$, and $\mathbf{H}=\frac{\mathbf{h}}{\sqrt{\mathbf{h}^{2}+\mathbf{x}^{2}+\mathbf{y}^{2}}}$

Then Equation (4) can be rewritten in the following simple formula

$$
\begin{gathered}
\varepsilon(P)=(H)^{m+3}=(F)^{m+3} \cdot(G)^{m_{l+3}} \\
\text { Or } \quad \log \varepsilon(P)=\left(m_{t}+3\right) \log F+\left(m_{l}+3\right) \log G .
\end{gathered}
$$

2.2. The Determination of the relative luminance $\boldsymbol{L}^{\prime}(\boldsymbol{P})$ at a point $\boldsymbol{P}$ on the road surface

The calculation of the relative luminance $\boldsymbol{L}^{\prime}(\boldsymbol{P})$ at a point $\boldsymbol{P}$ is made somewhat easier if the so-called reduced luminance coefficient is used which depends upon the light source and the observer relative to the element under consideration. The luminance at a point $\boldsymbol{p}$ on the road surface $\left(\boldsymbol{L}_{\boldsymbol{P}}\right)$ can be determined using the following formula [4].

$$
\mathrm{L}_{\mathrm{P}}=\mathrm{q} \cdot \mathrm{E}_{\mathrm{P}}
$$

Where $\mathrm{q}$ is the luminance coefficient; then from equation (2) we have

hence,

$$
\begin{gathered}
\mathrm{L}_{\mathrm{P}}=\mathrm{q}(\cos \alpha)^{3} \cdot(\cos \alpha)^{m} \mathrm{E}_{\mathrm{o}} \\
\mathrm{L}_{\mathrm{P}}=\mathrm{r} \cdot(\cos \alpha)^{m} \mathrm{E}_{\mathrm{o}} \\
\mathrm{r}=\mathrm{q}(\cos \alpha)^{3}
\end{gathered}
$$

where

Then from Equation (12), the maximum value of the luminance $L_{o}$ can be determined by the following:

$$
\mathrm{L}_{\mathrm{o}}=\mathrm{r}_{\mathrm{o}} \cdot \mathrm{E}_{\mathrm{o}}
$$

Where: $\mathbf{r}_{\mathbf{o}}$ is the value of $\mathbf{r}$ when $\alpha=0$; Then from equation (12) and (13) we have

$$
L^{\prime}(P)=\frac{L_{P}}{L_{o}}=\frac{r}{r_{o}} \cdot(\cos \alpha)^{m}
$$

Then from equation (3), $\mathrm{L}^{\prime}(\mathrm{P})$ can be given by the formula

Where $\rho=\frac{r}{r_{0}}$

$$
\mathrm{L}^{\prime}(\mathrm{P})=\rho \cdot \varepsilon(\mathrm{P}) \cdot(\cos \alpha)^{-3}
$$

Equation (15) gives $\mathrm{L}^{\prime}(\mathrm{P})$ as a function of the relative illuminance and the reduced luminance coefficient $(r)$ at the angels of light incidence zero and $\boldsymbol{\alpha}$. The values of $(\boldsymbol{r})$ at the points considered in this work are taken from $\boldsymbol{r}$ table of standard road surface $\boldsymbol{R}_{2}$ [4].

Note: The values of $(\boldsymbol{r})$ in such table depends on the angle $\boldsymbol{\alpha}$ and the angle between the plane of light incidence and the plane of observation $\beta$, [see Figure (2) and Table $1]$. 


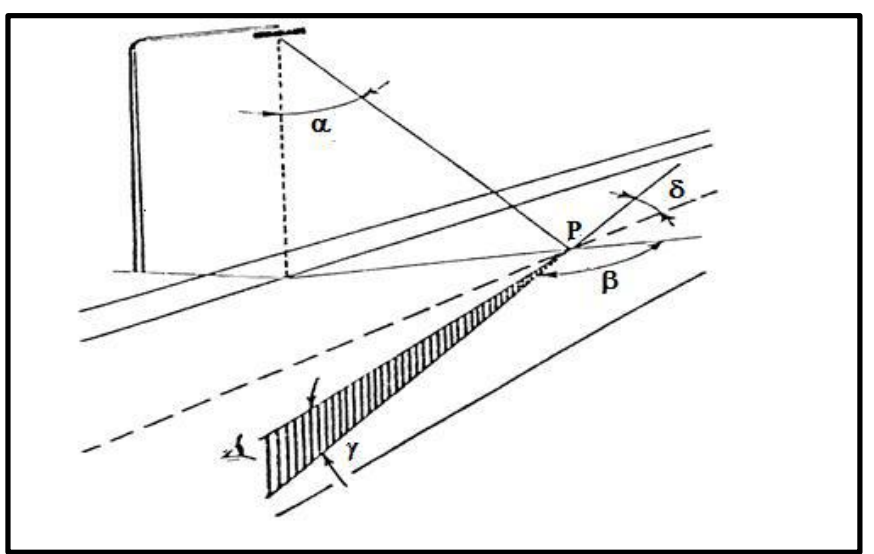

Fig. 2. The values of (r) in such table depends on the angle $\boldsymbol{\alpha}$ and the angle between the plane of light incidence and the plane of observation $\beta$.

Table 1. The values of the reduced luminance coefficient (r) at the considered points on the road surface in the figure (2) and the point $P$.

\begin{tabular}{lcccc}
\hline The points & $\mathbf{P}$ & $\left(\mathbf{W}_{\mathbf{1}}, \mathbf{O}\right)$ & $(\mathbf{O}, \mathbf{S})$ & $\left(\mathbf{W}_{\mathbf{1}}, \mathbf{S}\right)$ \\
\hline $\mathbf{r}$ at $\boldsymbol{\beta}_{1}=90^{\circ}$ & 0.1514 & 0.0985 & 0.0521 & 0.0321 \\
$\mathbf{r}$ at $\boldsymbol{\beta}_{2}=120^{\circ}$ & 0.1542 & 0.1392 & 0.075 & 0.0607 \\
\hline
\end{tabular}

\section{Calculations and Results}

In this work to take advantage of Equation (7) or (9) the calculation aimed to realize the following two important technical points.

\section{1. a-The isolux diagram calculation}

Let the arrangement of the roadway layout be as shown in Figure (1), where $\mathbf{O}$ is the origin, $\mathbf{x}$, and $\mathbf{y}$ are the axes of the coordinates. Hence, the roadway is represented as a part of the $(\mathrm{x}-\mathrm{y})$ plane bounded by $\mathrm{x}=\mathrm{W}_{1}$ on the roadside and $\mathrm{x}=\mathrm{W}_{2}$ on the kerb side. The road surface is assumed straight with infinite length, and width $\mathrm{W}_{1}=16$ $\mathrm{m}$. The luminaire is assumed to be installed at the kerb edge, and its overhang equals to $\mathrm{w}_{2}=2 \mathrm{~m}$, let $\mathbf{S}$ be half of the span between the two successive luminaires.

Note: the values of $\boldsymbol{m}_{t}$ and $\boldsymbol{m}_{l}$ corresponding to different values of $\mathbf{C F}$ are taken from literature [5] and represented in Table (2).

Table 2. The values of the relative illuminance of the point $\mathrm{P},\left(\mathrm{W}_{1}, \mathrm{O}\right),(\mathrm{O}, \mathrm{S})$, and $\left(\mathrm{W}_{1}, \mathrm{~S}\right)$.

\begin{tabular}{lrrrrrrr}
\hline $\mathbf{C . F}$ & $\mathbf{1 0}$ & $\mathbf{8}$ & $\mathbf{6}$ & $\mathbf{4}$ & $\mathbf{2}$ & $\mathbf{1 . 5}$ & $\mathbf{1 . 2}$ \\
\hline $\mathbf{L o g} \mathbf{C . F}$ & 1.0000 & 0.9031 & 0.7782 & 0.6021 & 0.3010 & 0.1761 & 0.0792 \\
$\mathbf{m}_{\mathfrak{t}} / \mathbf{m}_{\mathbf{l}}$ & 2.3256 & 2.1002 & 1.8100 & 1.4002 & 0.7001 & 0.4095 & 0.1841 \\
$\mathbf{m}_{\mathbf{t}}$ & 0.1163 & 0.1363 & 0.1563 & 0.1763 & 0.1963 & 0.2163 & 0.2363 \\
$\mathbf{m}_{\mathbf{l}}$ & 0.0500 & 0.0649 & 0.0864 & 0.1259 & 0.2804 & 0.5283 & 1.2835 \\
$\boldsymbol{\varepsilon}(\mathbf{P})$ & 0.2252 & 0.2234 & 0.2211 & 0.2175 & 0.2056 & 0.1881 & 0.1744 \\
$\boldsymbol{\varepsilon}\left(\mathbf{W}_{\mathbf{1}}, \mathbf{O}\right)$ & 0.1383 & 0.1366 & 0.1348 & 0.1331 & 0.1315 & 0.1298 & 0.1282 \\
$\boldsymbol{\varepsilon}(\mathbf{O}, \mathbf{S})$ & 0.0626 & 0.0617 & 0.0606 & 0.0584 & 0.0508 & 0.0405 & 0.0204 \\
$\boldsymbol{\varepsilon}\left(\mathbf{W}_{\mathbf{1}}, \mathbf{S}\right)$ & 0.0364 & 0.0358 & 0.0345 & 0.0336 & 0.0265 & 0.0232 & 0.0172 \\
\hline
\end{tabular}

Referring to equation (3) $\boldsymbol{\varepsilon}(\mathbf{P})$ may have the values $0.1,0.2, \ldots \ldots, 0.9$. 
Considering the case of a single luminaire having $\mathbf{C F}=10$ and $\mathbf{h}=10$ meters. Then, values of all $\boldsymbol{\varepsilon}(\mathbf{P})$, the variables in Equation (7) are given except the $\mathbf{x}$ and $\mathbf{y}$ coordinates of the points satisfying such equation. Assuming different values of $\mathbf{y}$ then from Equation (7) we obtain the corresponding values of $\mathbf{x}$. Then we have different points having the same value of $\boldsymbol{\varepsilon}(\mathbf{P})$. Let such points be represented in Figure(3), hence the curve joining such points represents the isolux contour corresponding to such constant value of $\boldsymbol{\varepsilon}(\mathbf{P})$. Repeating the above-mentioned procedure to obtain the isolux contours corresponding to the values of $\boldsymbol{\varepsilon}(\mathbf{P})$ between 0.1 and 0.9 , this constitutes the isolux diagram.

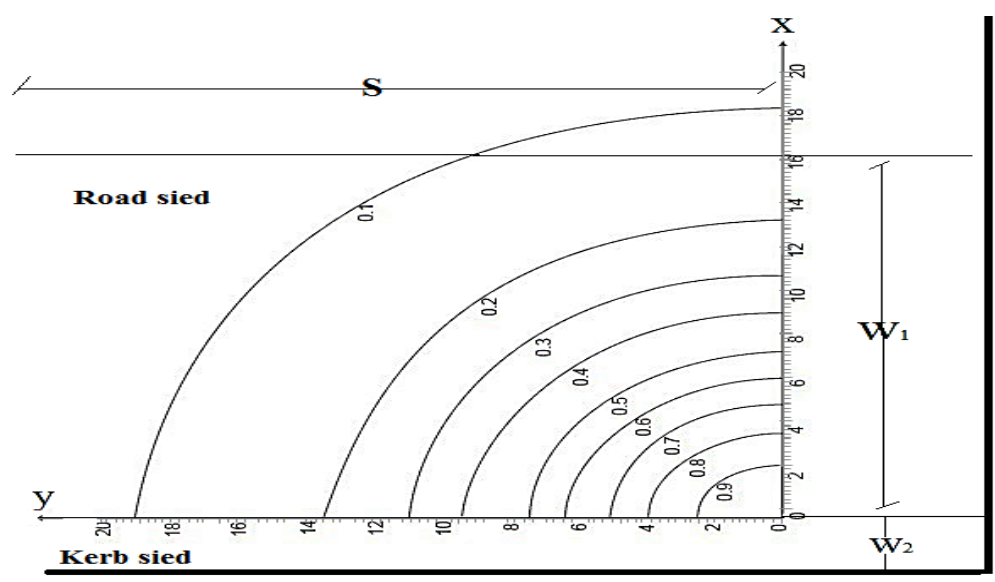

Fig. 3. The isolux diagram.

3.1-b. Calculating the relative illuminance and relative luminance of the road surface at any point on the road surface.

First -the calculation of $\varepsilon(\mathrm{P})$ :

Considering the layout represented in Figure (1) assuming that the coordinate of the point $\boldsymbol{A}, \boldsymbol{B}$, and $\boldsymbol{P}$, are $(8,0),(0,10)$, and $(8,10)$ respectively and $\boldsymbol{h}=10 \mathrm{~m}$ then from Equation (9) we have the values of $\boldsymbol{F}=0.87$ and $\boldsymbol{G}=0.707$. Then $\boldsymbol{\varepsilon}(\boldsymbol{p})$ can be obtained by substituting in equation (10), the values of for different values of $\mathbf{C F}$ are illustrated in Table (1)

Second- the calculation of $L^{\prime}(P)$ :

The value of $\mathbf{r}$ taken from standard road surface Table (2), then from equation (15) the value of $\boldsymbol{L}^{\prime}(\boldsymbol{P})$ can be calculated for different values of $\mathbf{C F}$, and the results are illustrated in Table (3).

Table 3. Values of the relative luminance of the points $\mathrm{P},\left(\mathrm{W}_{1}, \mathrm{O}\right),(\mathrm{O}, \mathrm{S})$, and $\left(\mathrm{W}_{1}, \mathrm{~S}\right)$ at different values of C.F.

\begin{tabular}{llclllll}
\hline C.F & \multicolumn{1}{c}{$\mathbf{1 0}$} & \multicolumn{1}{c}{$\mathbf{8}$} & \multicolumn{1}{c}{$\mathbf{6}$} & \multicolumn{1}{c}{$\mathbf{4}$} & \multicolumn{1}{c}{$\mathbf{2}$} & $\mathbf{1 . 5}$ & $\mathbf{1 . 2}$ \\
\hline Log C.F & 1 & 0.9031 & 0.7782 & 0.6021 & 0.301 & 0.1761 & 0.0792 \\
$\mathbf{L}^{\prime} \boldsymbol{\beta} \mathbf{1}(\mathbf{P})$ & 0.2624 & 0.2600 & 0.2574 & 0.2532 & 0.2393 & 0.21897 & 0.1681 \\
$\mathbf{L}^{\prime} \boldsymbol{\beta} \mathbf{2}(\mathbf{P})$ & 0.2670 & 0.2648 & 0.26213 & 0.25783 & 0.24372 & 0.223 & 0.1712 \\
$\mathbf{L}^{\prime} \boldsymbol{\beta} \mathbf{1}(\mathbf{W}, \mathbf{O})$ & 0.1642 & 0.1622 & 0.1602 & 0.1680 & 0.1561 & 0.1541 & 0.1522 \\
$\mathbf{L}^{\prime} \boldsymbol{\beta} \mathbf{2}(\mathbf{W}, \mathbf{O})$ & 0.2336 & 0.2307 & 0.2278 & 0.2248 & 0.2282 & 0.2192 & 0.21646 \\
$\mathbf{L}^{\prime} \boldsymbol{\beta} \mathbf{1}(\mathbf{O}, \mathbf{S})$ & 0.08936 & 0.0881 & 0.0865 & 0.08337 & 0.0725 & 0.0578 & 0.2912 \\
$\mathbf{L}^{\prime} \boldsymbol{\beta} \mathbf{2}(\mathbf{O}, \mathbf{S})$ & 0.1286 & 0.12679 & 0.1245 & 0.1200 & 0.1044 & 0.0832 & 0.04129 \\
$\mathbf{L}^{\prime} \boldsymbol{\beta} \mathbf{1}\left(\mathbf{W}_{\mathbf{1}}, \mathbf{S}\right)$ & 0.0539 & 0.053 & 0.05183 & 0.0498 & 0.03924 & 0.0343 & 0.02552 \\
$\mathbf{L}^{\prime} \boldsymbol{\beta} \mathbf{2}\left(\mathbf{W}_{\mathbf{1}}, \mathbf{S}\right)$ & 0.102 & 0.1003 & 0.0981 & 0.09416 & 0.07426 & 0.0649 & 0.03447 \\
\hline
\end{tabular}




\section{2-a. The dependence of the isolux-diagram features on the luminaire CF value}

As mentioned in (3-1-a) the values of the illuminance $\varepsilon\left(\boldsymbol{W}_{\boldsymbol{l}}, \boldsymbol{O}\right), \boldsymbol{\varepsilon}(\boldsymbol{O}, \boldsymbol{S})$, and $\varepsilon\left(W_{l}, S\right)$ are the isolux diagram features. It is clear that such features are dependent upon the geometrical relationship of the roadway and the lighting system and hence affecting the uniformity of both the illuminance and luminance on the road surface. The values of $\varepsilon\left(W_{l}, O\right), \varepsilon(O, S)$, and $\varepsilon\left(W_{l}, S\right)$ are obtained by subsiding $\mathrm{x}=\mathrm{W} 1=16$ $\mathrm{m}$ and $\mathrm{y}=\mathrm{S}=22.7 \mathrm{~m}$ in Equation (7), the obtained values are represented in Table (2). The luminance values corresponding to such factors are obtained by subsiding in equation (15) for (r) from the table (2) corresponding to $\boldsymbol{\beta}=90^{\circ}$ and $120^{\circ}$. The results of the calculated values for $\boldsymbol{\beta}=90$ and $\boldsymbol{\beta}=120$ are represented in Table (3).

\section{2-b- The calculation of the absolute illuminance and luminance uniformity}

Considering the roadway lighting system consisting of a single luminance as illustrated in Figure (2). The minimum values of both $(\boldsymbol{p})$, and $\boldsymbol{L}^{\prime}(\boldsymbol{p})$ are expected at the point $(\boldsymbol{W} \boldsymbol{1}, \boldsymbol{S})$. Hence, according to the definitions of both the absolute illuminance $\boldsymbol{U} \boldsymbol{i}$ and luminance $\boldsymbol{U} \boldsymbol{l}$ uniformities mentioned in the previous section (I.4), we have $\boldsymbol{U}_{\boldsymbol{i}}$ $=\varepsilon(W 1, S)$ which can be calculated by substituting $\mathbf{x}=\mathbf{W 1} \& \mathbf{y}=\mathbf{S}$ in Equation (7). Hence, it can be calculated by substituting the obtained values of $\boldsymbol{\varepsilon}(\boldsymbol{W l}, \boldsymbol{S})$ in Equation (15).

Note: $\boldsymbol{L}^{\prime}(\boldsymbol{W} \boldsymbol{1}, \boldsymbol{S})$ may have corresponding to different values of the angle $\boldsymbol{\beta}_{\boldsymbol{1}}$. In this work, we consider the values of $\beta_{1}=90^{\circ}$ and $\beta_{1=120^{\circ}}$.

\section{Conclusion}

1- The derived equation (Equation 15) enables us to draw the isolux diagram on the road lighting system knowing the indices of the luminaire lighting distribution across and along the road surface, the luminaire mounting height, the road width, and the spacing between the luminaires.

2- The obtained values of $\boldsymbol{\varepsilon}(\mathbf{P})$ at the three points determined the isolux diagram features and showed that $\varepsilon\left(\mathbf{W}_{1}, \mathbf{O}\right)>\varepsilon(\mathbf{O}, \mathbf{S})>\varepsilon\left(\mathbf{W}_{\mathbf{1}}, \mathbf{S}\right)$. Then the minimum illuminance on the road surface is the value of $\varepsilon\left(\mathbf{W}_{1}, \mathbf{S}\right)$ which enables the prediction of the uniformities of both illuminance and luminance, their average values and hence the effectiveness and suitability of the lighting system.

3- Since $\mathbf{S}$ is the half of the span between two successive luminaires. The value of $\boldsymbol{\varepsilon}$ $\left(\mathbf{W}_{1}, \mathbf{S}\right)$ represented in Table (1) is due to a single luminaire. Thus the real value of $\varepsilon\left(\mathbf{W}_{1}, \mathbf{S}\right)$ is twice the value in Table (1) which equals to 0.0724. Usual roadway lighting practice calls for a max-to- min ratio $12: 1$ or less i.e. the minimum value of the relative illuminance must be 0.083 or less [8]. This means that the obtained results in this work are nearly agreeing with I.E.S standard practice.

4- The results illustrated in Tables (2) and (3) show that the luminaires having higher CF values are the better.

\section{References}

1. Ewing, J. L. and Keck, M. E. Street lighting luminaire distributions for uniform luminance and Illumination. Journal of IESP (190) / APRIL (1978).

2. VanBommel, W. J. M. Design considerations by road lighting. Journal, IESP (40) / October (1978). 
3. Meshko, V. V. V. Fundamental of illuminating engineering. Mir publication, Moscow, P (25), (1981).

4. Van Bommel, W. J. M., Boe, J. B. Road lighting "Philips technical library Eindhoven, P (119-169) May (1980).

5. Safaa, El Mahy and EL-Ganainy, M. M. Optimization of light distribution (LD) for road lighting luminaires of different cross factors (C.F). Advanced in Environmental Biology,10 (7), P (62 -67) July (2016).

6. El-Ganainy, M. M., Ibrahim, M. M., Abdel Hamied, Ma., and El- kaream, S. M. The dependence of 1solux Diagram Pattern on the Parameter (m) of Exterior Distribution. Bull. N.R. C., Egypt, Vol 23, Nol. (89- 96). 1998.

7. Knorring, G.M. Illuminating Engineering calculation for artificial lighting. $p$ (13-14) (24 - 25), Energia (1973).

8. Charle, H. Loch. Roadway lighting calculations in perspective. Journal of IES. Page (663-669) April, (1985). 